\title{
Nonadditive entropy reconciles the area law in quantum systems with classical thermodynamics
}

\author{
Filippo Caruso ${ }^{1}$ and Constantino Tsallis ${ }^{2,3}$ \\ 1 NEST CNR-INFM and Scuola Normale Superiore, Piazza dei Cavalieri 7, I-56126 Pisa, Italy \\ 2 Centro Brasileiro de Pesquisas Fisicas, Rua Xavier Sigaud 150, 22290-180 Rio de Janeiro, Brazil \\ ${ }^{3}$ Santa Fe Institute, 1399 Hyde Park Road, Santa Fe, New Mexico 87501, USA
}

\begin{abstract}
The Boltzmann-Gibbs-von Neumann entropy of a large part (of linear size $L$ ) of some (much larger) $d$-dimensional quantum systems follows the so-called area law (as for black holes), i.e., it is proportional to $L^{d-1}$. Here we show, for $d=1,2$, that the (nonadditive) entropy $S_{q}$ satisfies, for a special value of $q \neq 1$, the classical thermodynamical prescription for the entropy to be extensive, i.e., $S_{q} \propto L^{d}$. Therefore, we reconcile with classical thermodynamics the area law widespread in quantum systems. Recently, a similar behavior was exhibited, by M. Gell-Mann, Y. Sato and one of us (C.T.), in mathematical models with scale-invariant correlations. Finally, we find that the system critical features are marked by a maximum of the special entropic index $q$.
\end{abstract}

PACS numbers: 05.70.Jk, 05.30.-d

The aim of statistical mechanics is to establish a direct link between mechanical microscopic laws and classical thermodynamics. The most famous classical theory in this field was developed by Boltzmann and Gibbs (BG) and it is considered as one of the cornerstones of contemporary physics. The connection between the microand macro-world is usually described by the so-called BG entropy,

$$
S_{B G}=-k \sum_{i=1}^{W} p_{i} \ln p_{i},
$$

where $k$ is a positive constant, $W$ is the number of microscopic states, and $\left\{p_{i}\right\}_{i=1, \ldots, W}$ is a normalized probability distribution. The subtle concept of entropy lays the foundation of classical thermodynamics. The BG entropy is additive, i.e. $S_{B G}(A, B)=S_{B G}(A)+S_{B G}(B)$, where $A$ and $B$ are two probabilistically independent subsystems. One of the crucial properties of the entropy in the context of classical thermodynamics is extensivity, namely proportionality with the number of elements of the system, when this number is large. The BG entropy satisfies this prescription if the subsystems are statistically (quasi)independent, or typically if the correlations within the system are generically local. In such cases the system is called extensive.

In general, however, the situation is not always of this type and correlations may be far from negligible at all scales. In such cases the BG entropy (of the entire system or large part of it) may be nonextensive. Nonetheless, for an important class of such systems, an entropy exists that is extensive in terms of the microscopic probabilities [1]. The additive BG entropy can be generalized into the nonadditive $q$-entropy [2, 3]

$$
S_{q}=k \frac{1-\sum_{i=1}^{W} p_{i}^{q}}{q-1}, \quad q \in \mathcal{R} \quad\left(S_{1}=S_{B G}\right) .
$$

This is the basis of the so-called nonextensive statistical mechanics [4, 5] (see [46] for a regularly updated bibliography), which generalizes the BG theory. In the context of cybernetics and information theory, the same type of entropic form has been advanced in [6].

Additivity (for two probabilistically independent subsystems $A$ and $B$ ) is generalized by the following pseudoadditivity: $\quad S_{q}(A, B) / k=S_{q}(A) / k+S_{q}(B) / k+(1-$ q) $S_{q}(A) S_{q}(B) / k^{2}$. For subsystems that have special probability correlations, extensivity is not valid for $S_{B G}$, but may occur for $S_{q}$ with a particular value of the index $q \neq 1$, called the $q$-entropic index $q_{\text {ent }}$. Such systems are sometimes referred to as nonextensive [1, 5]. The physical relevance of the $q$-entropy is related also to the recent generalization of the central limit theorem (q-CLT), where $q$-Gaussian distributions take the place of the usual Gaussians when considering systems with strong correlations [7]. Much experimental evidence of predictions of nonextensive statistical mechanics is available: see, for instance, 8, 9]. Let us emphasize the difference between additivity and extensivity for the entropy. Additivity depends only on the mathematical definition of the entropy; therefore, $S_{1}$ is additive, whereas $S_{q}(q \neq 1)$ is nonadditive. Extensivity is more subtle, since it also depends on the specific system, as we will show in this paper.

A physical system may exhibit genuine quantum aspects. In particular, quantum correlations, quantified by entanglement, can be present. The classical probability concepts are replaced by the density matrix operator $\hat{\rho}$, in a more general probability amplitude context. The quantum counterpart of the BG entropy, which is called the von Neumann entropy, is thus given by $S_{1}(\hat{\rho})=-k \operatorname{Tr} \hat{\rho} \ln \hat{\rho}$, while the classical $q$-entropy is replaced by:

$$
S_{q}(\hat{\rho})=k \frac{1-\operatorname{Tr} \hat{\rho}^{q}}{q-1}
$$

The pseudo-additivity is now given by

$$
\frac{S_{q}\left(\hat{\rho}_{1} \otimes \hat{\rho}_{2}\right)}{k}=\frac{S_{q}\left(\hat{\rho}_{1}\right)}{k}+\frac{S_{q}\left(\hat{\rho}_{2}\right)}{k}+(1-q) \frac{S_{q}\left(\hat{\rho}_{1}\right)}{k} \frac{S_{q}\left(\hat{\rho}_{2}\right)}{k},
$$

from now on $\mathrm{k}=1$. 
In the following we will analyze fermionic and bosonic quantum systems in which strong non-classical correlations exist between their components. The appearance of long-range correlations in the ground state of a quantum many-body system, undergoing, for instance, a quantum phase transition at zero temperature, is deeply related to entanglement [10]. Quantum spin chains, composed of a set of localized spins coupled through exchange interaction in an external transverse magnetic field, capture the essence of these intriguing phenomena and have been extensively studied [11, 12, 13, 14, 15, 16]. The degree of entanglement between a block of $L$ contiguous spins and the rest of the chain in its ground state is measured by the von Neumann entropy of the block. For large block size, it typically saturates off criticality, whereas it is logarithmically unbounded at the critical point.

Here we show that the nonadditive entropy [2, 5] $S_{q}\left(\hat{\rho}_{L}\right) \equiv k \frac{1-\operatorname{Tr} \hat{\rho}_{L}^{q}}{q-1}, \hat{\rho}_{L}$ being the density matrix of a block of $L$ spins of the ground state of quantum spin chains in the neighborhood of a quantum phase transition, is extensive [i.e., $S_{q}\left(\hat{\rho}_{L}\right) \propto L$ for $L \gg 1$ ] for special values of $q<1$. The additive von Neumann entropy $S_{1}\left(\hat{\rho}_{L}\right)=-k \operatorname{Tr} \hat{\rho}_{L} \ln \hat{\rho}_{L}$ is nonextensive [indeed, $\lim _{L \rightarrow \infty} S_{1}\left(\hat{\rho}_{L}\right) / L=0$ ] in all considered cases; the same happens with the additive Renyi entropy.

A similar behavior can be observed for another important class of quantum systems, harmonic lattice Hamiltonians, i.e., bosons interacting through a quadratic Hamiltonian in dimension $d=2$. These systems are discrete versions of the free scalar Klein-Gordon field. The degree of entanglement between a square block of $L^{2}$ oscillators and its exterior, as measured by the additive von Neumann entropy of the square block, $S_{1}\left(\hat{\rho}_{L^{2}}\right)$ is linear in $L$ (area law), i.e. it is nonextensive [17, 18, 19, 20, 21]. Here we show that the nonadditive entropy $S_{q}\left(\hat{\rho}_{L^{2}}\right)$ is extensive [i.e., $S_{q}\left(\hat{\rho}_{L^{2}}\right) \propto L^{2}$ for $L \gg 1$ ] for special values of $q<1$.

Therefore, we present here two physical realizations, in many-body Hamiltonian systems, of the abstract mathematical examples recently exhibited by M. Gell-Mann, Y. Sato and one of us (C.T.) in Ref. [1], that, for anomalous values of $q$, the nonadditive entropy $S_{q}$, can be extensive, as expected from the Clausius thermodynamical requirement for the entropy. In other terms, we show explicitly the first two physical examples of the fact that the nonadditive entropy reconciles the area law (e.g., typical of black holes) with classical thermodynamics. In addition to the considerable advantage of $S_{q}$ enabling thus the use of all standard thermodynamical relations, this constitutes a powerful tool to detect strong nonlocal correlations in quantum many-body systems, by a nonadditive measure [22, 23, 24, 25, 26, 27, 28]. Indeed, this entropic index $q$ presents a maximum when the correlation length is divergent in the ground state of these quantum systems.

\section{QUANTUM SPIN CHAINS}

First of all, we focus our investigations on a onedimensional spin-1/2 ferromagnetic chain with an exchange (local) coupling and subjected to an external transverse magnetic field, i.e., the quantum XY model. The Hamiltonian is given by:

$\hat{\mathcal{H}}=-\sum_{j=1}^{N-1}\left[(1+\gamma) \hat{\sigma}_{j}^{x} \hat{\sigma}_{j+1}^{x}+(1-\gamma) \hat{\sigma}_{j}^{y} \hat{\sigma}_{j+1}^{y}+2 \lambda \hat{\sigma}_{j}^{z}\right]$

where $\hat{\sigma}_{j}^{\alpha}(\alpha=x, y, z)$ are the Pauli matrices of the $j$-th spin, $N$ is the number of spins of the chain, and $\gamma$ and $\lambda$ characterize, respectively, the strength of the anisotropy parameter and of a trasverse magnetic field along the $z$ direction. This model for $0<|\gamma| \leq 1$ belongs to the Ising universality class and it actually reduces to the quantum Ising chain for $|\gamma|=1$. At $T=0$, this system undergoes a quantum phase transition at the critical point $\left|\lambda_{c}\right|=1$ in the thermodynamic limit $N \rightarrow \infty$. For $\gamma=0$ it is the isotropic XX model, which is critical for $|\lambda| \leq 1[10]$.

Entanglement in the neighborhood of the quantum phase transition has been recently widely investigated 11, 12, 13, 14, 15, 16]. In particular it has been shown that one-site and two-site entanglement between nearest or next-to-nearest spins display a peak near or at the critical point [11, 12]. Moreover, the entanglement between a block of $L$ contiguous spins and the rest of the chain in the ground state, quantified by the von Neumann entropy, presents a logarithmic divergence with $L$ at criticality, while it saturates in a non-critical regime [13, 14, 15].

The inadequacy of the additive von Neumann entropy as a measure of the information content in a quantum state was pointed out in Ref. [23]. A theoretical observation that the measure of quantum entanglement may not be additive has been discussed in Refs. [22, 23, 24, 25, 26, 27, 28]. Recently, Ref. [29] suggested to abandon the a priori probability postulate going beyond the usual BG situation.

Here we propose to extend the definition of the von Neumann entropy to a wider class of entropy measures (see also Ref. [30]) which naturally include it, thus generalizing the notion of the block entanglement entropy. The block $q$-entropy of a block of size $L$ is simply defined as the $q$-entropy, Eq. (3), of the reduced density matrix $\hat{\rho}_{L}$ of the block, $S_{q}\left(\hat{\rho}_{L}\right)$, when the total chain is in the ground state. In the following we show that, in contrast to the von Neumann entropy, there exists a $q$ value for which $S_{q}\left(\hat{\rho}_{L}\right)$ is extensive. This value does depend on the critical properties of the chain and it is consistent with the universality hypothesis.

The XY model in Eq. (4) can be diagonalized exactly with a Jordan-Wigner transformation, followed by a Bogoliubov rotation [15, 31, 32, 33]; this allows one to analytically evaluate the spectrum of $\hat{\rho}_{L}$ in the thermodynamic limit $N \rightarrow \infty$. More details are shown also in Ref. [34. 


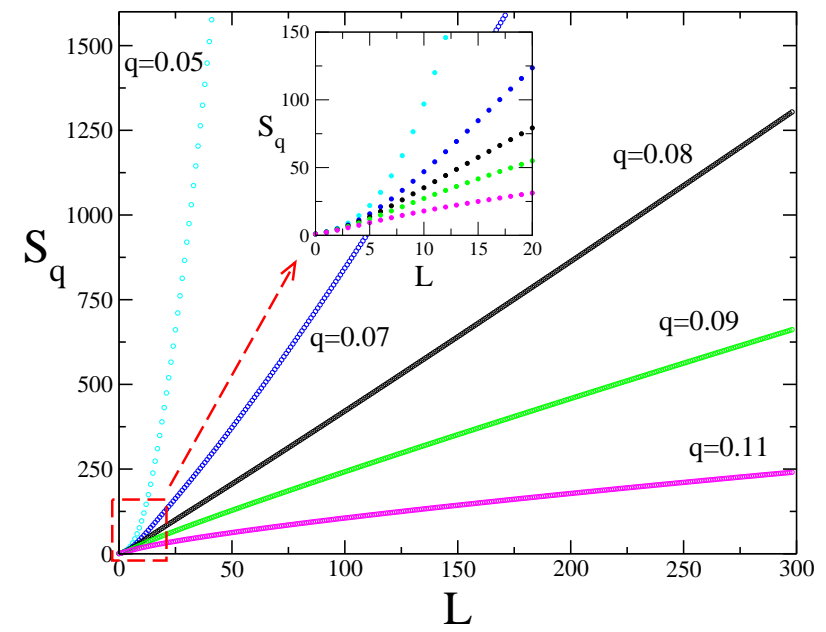

FIG. 1: Block $q$-entropy $S_{q}\left(\hat{\rho}_{L}\right)$ as a function of the block size $L$ in a critical Ising chain $(\gamma=1, \lambda=1)$, for typical values of $q$. Only for $q=q_{\text {ent }} \simeq 0.0828$ is $s_{q}$ finite (i.e., $S_{q}$ is extensive); for $q<q_{\text {ent }}\left(q>q_{\text {ent }}\right)$ it diverges (vanishes).

We first analyze the anisotropic quantum XY model, Eq. (4) with $\gamma \neq 0$, which has a critical point at $\lambda_{c}=1$. The block $q$-entropy as a function of the block size can show completely different asymptotic behaviors, when the entropic index $q$ is varied. In particular, here we are interested in a thermodynamically relevant quantity, namely the slope, denoted $s_{q}$, of $S_{q}$ versus $L$. It is generically not possible to have a finite value of $s_{1}$ : the entanglement entropy, evaluated by the von Neumann entropy, either saturates or diverges logarithmically in the thermodynamic limit, for respectively non-critical or critical spin chains [13, 14, 15]. The situation dramatically changes when the q-entropy formula in Eq. (3) is used: qualitatively it happens that, regardless the presence or absence of criticality, a $\lambda$-dependent value of $q$, denoted $q_{\text {ent }}$, exists such that, in the range $1 \ll L \ll \xi$ ( $\xi$ being the correlation length), $s_{q_{\text {ent }}}$ is finite, whereas it vanishes (diverges) for $q>q_{\text {ent }}\left(q<q_{\text {ent }}\right)$. We note that here the nonextensivity (i.e., $q \neq 1$ ) features are not due to the presence of say long-range interactions [35, 36] but they are triggered only by the fully quantum nonlocal correlations. In Fig. 1 we show, for the critical Ising model $(\gamma=1, \lambda=1)$, the behavior of the block $q$-entropy with respect to the block size: $S_{q}\left(\hat{\rho}_{L}\right)$ becomes extensive [i.e., $0<\lim _{L \rightarrow \infty} S_{q}\left(\hat{\rho}_{L}\right) / L<\infty$ ] at a $q$-entropic index $q_{\text {ent }} \simeq 0.0828 \pm 10^{-4}$ (with a corresponding entropic density $s_{q_{\text {ent }}} \approx 3.56 \pm 0.03$ ), thus satisfying the prescriptions of classical thermodynamics.

A very similar behavior is shown for the non-critical Ising model, as well as for critical and non-critical XY models with $0<\gamma<1$. The value of $q_{\text {ent }}$, for which $S_{q}\left(\hat{\rho}_{L}\right)$ is asymptotically extensive, is obtained by maximizing numerically the linear correlation coefficient $r$ of $S_{q}\left(\hat{\rho}_{L}\right)$, in the range $1 \ll L \ll \xi$, with respect to $q$, as shown in the inset in Fig. 2. Let us stress that, at

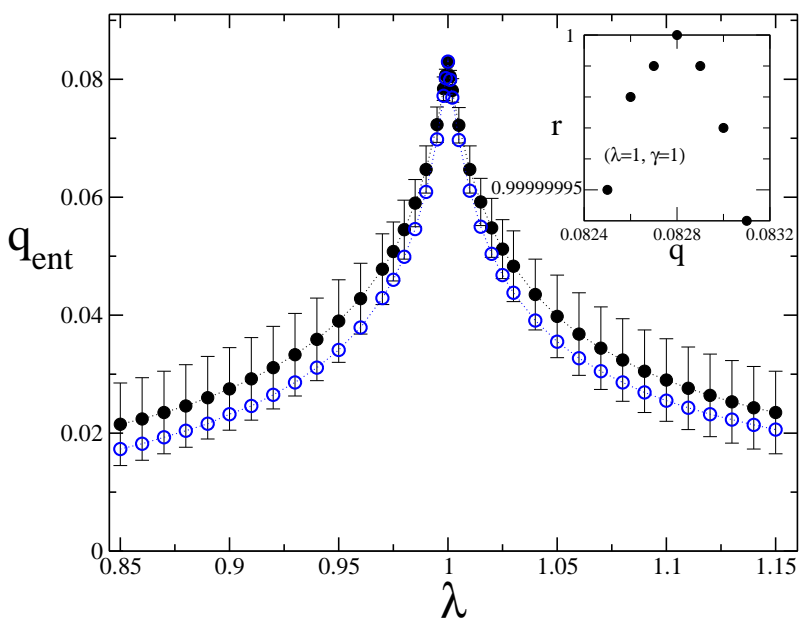

FIG. 2: The $\lambda$-dependence of the index $q_{\text {ent }}$ in the Ising $(\gamma=$ 1 , solid circle) and XY ( $\gamma=0.75$, empty circle $)$ chains. Inset: determination of $q_{\text {ent }}$ through numerical maximization of the linear correlation coefficient $r$ of $S_{q}\left(\hat{\rho}_{L}\right)$. The error bars for the Ising chain are obtained considering the variation of $q_{\text {ent }}$ when using the range $100 \leq L \leq 400$ in the search for $S_{q}\left(\hat{\rho}_{L}\right)$ linear behavior. At the present numerical level, a vanishing $q$ cannot be excluded off-criticality because of finite-size effects.

precisely the critical point, $\xi$ diverges, hence $L$ is unrestricted and can run up to infinity. The index $q_{\text {ent }}$ depends on the distance from criticality and it increases as $\lambda$ approaches $\lambda_{c}$ (Fig. (2). It is worth stressing that our numerical results satisfy the duality symmetry $\lambda \longrightarrow 1 / \lambda$, investigated in Ref. [37]. For the sake of clarity, notice that the value of $q_{\text {ent }}$ may be vanishing off criticality and this could not be found numerically because of the presence of finite-size effects. We have also checked other values of $\gamma$ for the XY model and the results are very similar to those presented here. This fact is consistent with the universality hypothesis. On one hand, the XY and Ising models (Ising universality class) have the same behavior as regards the extensivity of $S_{q}\left(\hat{\rho}_{L}\right)$. In Fig. 3 we report the variation of $s_{q_{e n t}}$ with respect to $\lambda$. On the other hand, for the isotropic XX model $(\gamma=0)$ in the critical region $|\lambda| \leq 1$ we find $q_{\text {ent }} \simeq 0.15 \pm 0.01$ for which $S_{q}\left(\hat{\rho}_{L}\right)$ becomes extensive.

Reference [38] enables us to analytically confirm (only at the critical point) our numerical results. The continuum limit of a $(1+1)$-dimensional critical system is a conformal field theory with central charge $c$. In this quite different context, the authors re-derive the result $S_{1}\left(\hat{\rho}_{L}\right) \sim(c / 3) \ln L$ for a finite block of length $L$ in an infinite critical system. To obtain the von Neumann entropy, they find an analytical expression for $\operatorname{Tr} \hat{\rho}_{L}^{q}$, namely $\operatorname{Tr} \hat{\rho}_{L}^{q} \sim L^{-c / 6(q-1 / q)}$. Here, we use this expression quite differently. We impose the extensivity of $S_{q}\left(\hat{\rho}_{L}\right)$ and we find the value of $q$ for which $-c / 6\left(q_{\text {ent }}-1 / q_{\text {ent }}\right)=1$, i.e.,

$$
q_{\text {ent }}=\frac{\sqrt{9+c^{2}}-3}{c} ;
$$




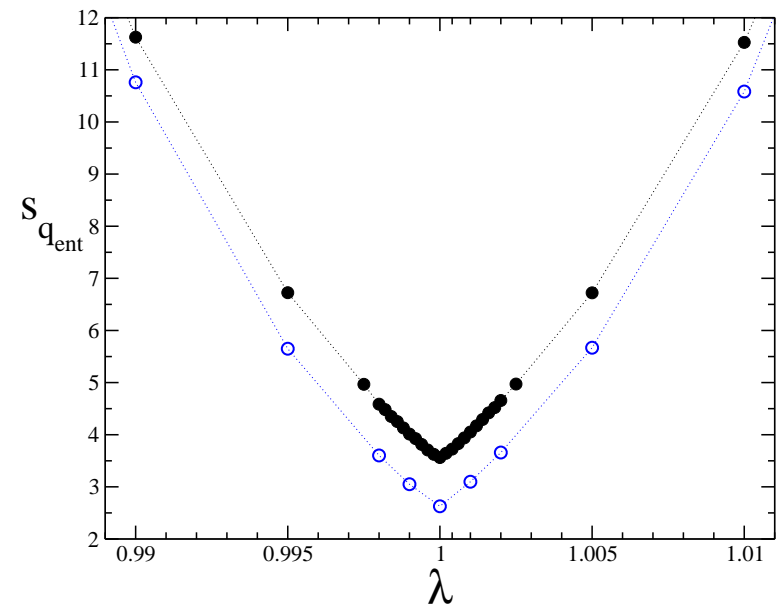

FIG. 3: $\lambda$-dependence of the $q$-entropic density $s_{q_{e n t}}$ in the Ising $(\gamma=1$, solid circle) and XY $(\gamma=0.75$, empty circle $)$ models. For $\lambda=1$, the slopes are 3.56 and 2.63, for $\gamma=1$ and $\gamma=0.75$, respectively. Notice the considerable variation in the values of the slope for even slightly different values of $\lambda$ out of criticality.

consequently, $\lim _{L \rightarrow \infty} S_{\underline{\sqrt{9+c^{2}-3}}}\left(\hat{\rho}_{L}\right) / L<\infty$. When $c$ increases from 0 to infinity (see Fig. (4), $q_{\text {ent }}$ increases from 0 to unity (von Neumann entropy). It is well known that for the critical quantum Ising and XY models the central charge is equal to $c=1 / 2$ (indeed they are in the same universality class and can be mapped to a free fermionic field theory). For these models, at $\lambda=1$, the value of $q$ for which $S_{q}\left(\hat{\rho}_{L}\right)$ is extensive is given by $q_{\text {ent }}=\sqrt{37}-6 \simeq 0.0828$, in perfect agreement with our numerical results in Fig. 2] The critical isotropic XX model $(\gamma=0$ and $|\lambda| \leq 1)$ is, instead, in another universality class, the central charge is $c=1$ (free bosonic field theory) and $S_{q}\left(\hat{\rho}_{L}\right)$ is extensive for $q_{\text {ent }}=\sqrt{10}-3 \simeq 0.16$, as found also numerically. Therefore, the universal behavior of the $q$-entropic index $q_{\text {ent }}$ is strictly related to the universal role played by the central charge in conformal field theory. Eq. (5) represents an additional connection between nonextensive statistical mechanical concepts and BG statistical mechanics at criticality. See Ref. 39] for another connection, where once again we verify that the $q$-entropic index typically characterizes universality classes. Let us note that, when the critical one-dimensional (1D) system is a semi-infinite chain, one has to replace $c$ with $c / 2$ in Eq. (5) [38].

It is worth mentioning that the Renyi entropy of a block of critical XX spin chains has been derived analytically in Refs. 40, 41]. Since the Renyi entropy is simply connected to the $q$-entropy, it is possible to rederive $q_{\text {ent }}$ for the critical XX model also from that analytical expression. Finally, let us point out that the reduction of the pure ground state of the full chain (at $T=0)$ to a finite block of $L$ spins results in a mixed state with quantum fluctuations. A mapping of this subsystem within a zero-temperature XX infinite chain to a

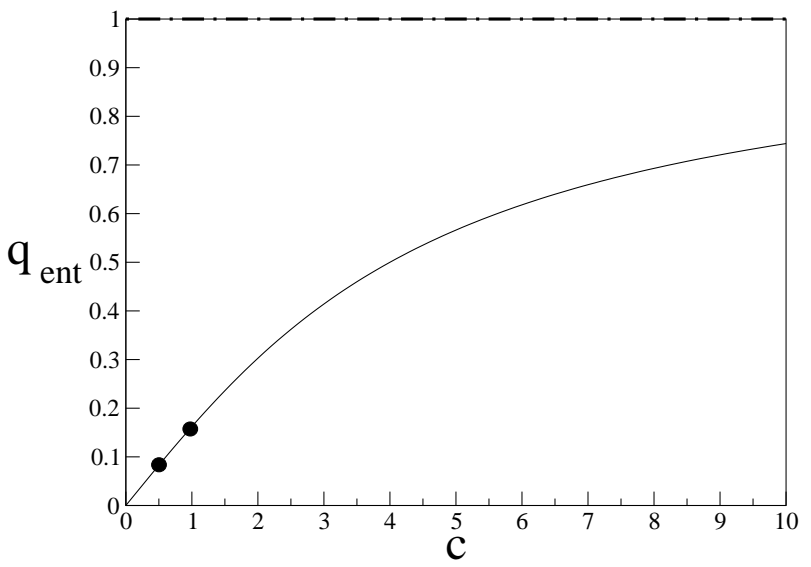

FIG. 4: $q_{\text {ent }}$ versus $c$ with the $q$-entropy, $S_{q}\left(\hat{\rho}_{L}\right)$, being extensive, i.e., $\lim _{L \rightarrow \infty} S_{\frac{\sqrt{9+c^{2}}-3}{c}}\left(\hat{\rho}_{L}\right) / L<\infty$. When $c$ increases

from 0 to infinity, $q_{\text {ent }} \stackrel{c}{\text { increases from } 0}$ to unity (von Neumann entropy). For the critical quantum Ising and XY models $c=1 / 2$ and $q_{\text {ent }}=\sqrt{37}-6 \simeq 0.0828$, while for the critical isotropic XX model $c=1$ and $q_{\text {ent }}=\sqrt{10}-3 \simeq 0.16$.

finite system which is thermalized at some finite temperature has been recently exhibited [42], thus defining an $L$ dependent effective temperature of the block. The use of a non-Boltzmannian distribution (e.g., the one emerging within nonextensive statistical mechanics) might enable the definition of an effective temperature which would not depend on $L$, as physically desirable. Indeed, this approach has been successfully implemented for $e-e^{+}$ collision experiments [43].

\section{2D BOSONIC SYSTEMS}

Now we present a second physical realization of the extensivity of $S_{q}$ in a bosonic $2 \mathrm{D}$ system at $T=0$. We start from a bidimensional (square lattice) system of infinite coupled harmonic oscillators studied in Ref. [17], with Hamiltonian

$H=\frac{1}{2} \sum_{x, y}\left[\Pi_{x, y}^{2}+\omega_{0}^{2} \Phi_{x, y}^{2}+\left(\Phi_{x, y}-\Phi_{x+1, y}\right)^{2}+\left(\Phi_{x, y}-\Phi_{x, y+1}\right)^{2}\right]$,

where $\Phi_{x, y}, \Pi_{x, y}$ and $\omega_{0}$ are the coordinate, momentum and self-frequency of the oscillator at site $\vec{r}=$ $(x, y)$. The system has the dispersion relation $E(\vec{k})=$ $\sqrt{\omega_{0}^{2}+4 \sin ^{2} k_{x} / 2+4 \sin ^{2} k_{y} / 2}$, i.e., a gap $\omega_{0}$ at $\vec{k}=$ $\overrightarrow{0}$. Applying the canonical transformation $b_{\mathbf{i}}=\sqrt{\frac{\omega}{2}} \times$ $\left(\Phi_{\mathbf{i}}+\frac{i}{\omega} \Pi_{\mathbf{i}}\right)$ with $\omega=\sqrt{\omega_{0}^{2}+4}$ and $i=1, \ldots, L^{2}$, the Hamiltonian in Eq. (6) is mapped to the quadratic canonical form

$$
H=\sum_{i j}\left[a_{i}^{\dagger} A_{i j} a_{j}+\frac{1}{2}\left(a_{i}^{\dagger} B_{i j} a_{j}^{\dagger}+\text { h.c. }\right)\right],
$$

where $a_{i}$ are bosonic operators and $i, j$ range from 1 to $L^{2}$. In Ref. [17] the authors find an asymptotic linear be- 


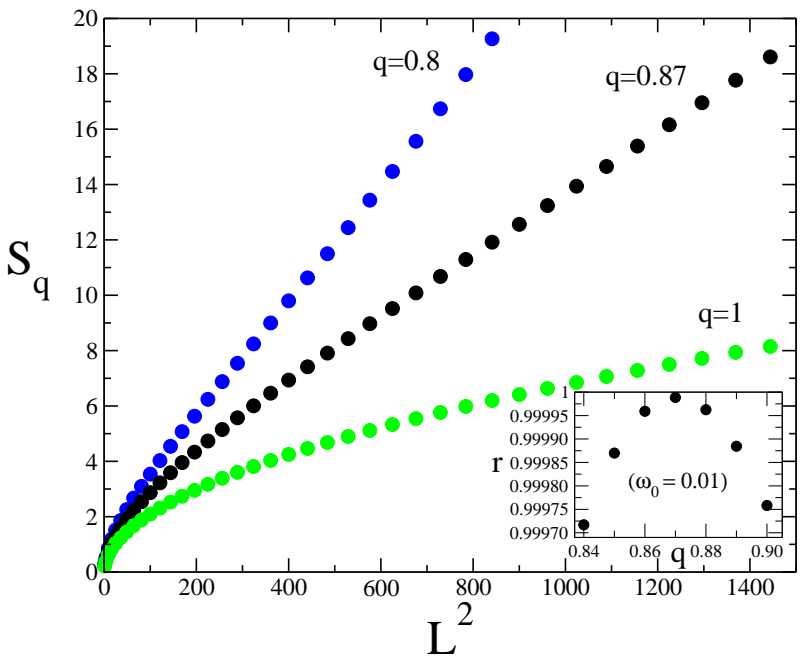

FIG. 5: Block $q$-entropy $S_{q}\left(\hat{\rho}_{L}\right)$ as a function of the square block area $L^{2}$ in a bosonic $2 \mathrm{D}$ array of infinite coupled harmonic oscillators at $T=0$, for typical values of $q$. Only for $q=q_{\text {ent }} \simeq 0.87, s_{q}$ is finite (i.e., $S_{q}$ is extensive); for $q<q_{\text {ent }}$ $\left(q>q_{\text {ent }}\right)$ it diverges (vanishes). Inset: determination of $q_{\text {ent }}$ through numerical maximization of the linear correlation coefficient $r$ of $S_{q}\left(\hat{\rho}_{L}\right)$ in the range $400 \leq L^{2} \leq 1600$.

havior (i.e., area law) of the block entanglement entropy as a function of the linear size $L$ (they consider square blocks of area $\left.L^{2}\right)$, for several $\omega_{0}\left(\lim _{L \rightarrow \infty} S_{1}\left(\hat{\rho}_{L}\right) / L^{2}=\right.$ $0)$, no matter how close the gap energy is to zero.

Here we study, instead, the behavior of the block $q$ entropy of the reduced density operator of a square block as a function of its area $L^{2}$, when the bosonic infinite two-dimensional system is in its ground state. We follow a similar procedure to the one used above for quantum spin chains (see Ref. [17] for more details). In Fig. [5 we show, in the case of $\omega_{0}=0.01$, that $S_{q}\left(\hat{\rho}_{L}\right)$ becomes extensive (i.e., $0<\lim _{L \rightarrow \infty} S_{q}\left(\hat{\rho}_{L}\right) / L^{2}<\infty$ ) at an index $q_{\text {ent }} \simeq 0.87$ (with a corresponding entropic density $s_{q_{e n t}} \approx 0.011$; see inset in Fig. (6). A very similar behavior is shown for other values of the gap. Let us point out that, unlike the linear behavior (for any gap energy) of the von Neumann entropy, now the index $q_{\text {ent }}$ depends on the gap and therefore measures the presence of a progressively divergent correlation length, as shown in Fig. 6.

\section{FINAL REMARKS}

We present two quantum many-body Hamiltonian physical realizations of the mathematical probabilistic models with scale-invariant correlations recently shown by M. Gell-Mann, Y. Sato and one of us (C.T.) in Ref. [1], in which the nonadditive entropy $S_{q}$ can be applied successfully (i.e., satisfying the classical thermodynamic requirement of extensivity). In this basic manner, we reconcile the entropy area law characterizing many quantum systems with classical thermodynamics. In addition

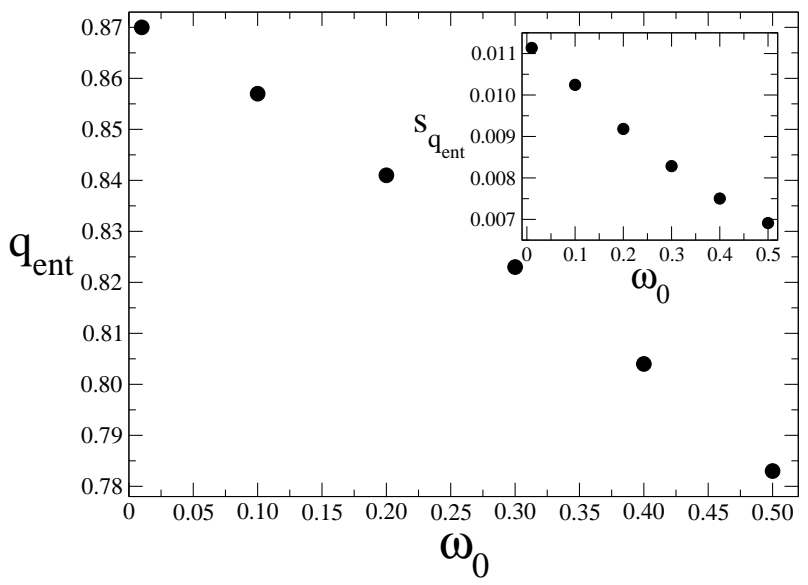

FIG. 6: $\omega_{0}$-dependence of the index $q_{\text {ent }}$ in a bosonic $2 \mathrm{D}$ array of infinite coupled harmonic oscillators at $T=0$. Inset: the $\omega_{0}$-dependence of the $q$-entropic density $s_{q_{e n t}}$.

to that, the present results show clearly the difference between additivity and extensivity for the entropy. Additivity depends only on the mathematical features of the entropy, e.g, $S_{1}$ is additive while $S_{q}(q \neq 1)$ is nonadditive. Extensivity is a more subtle concept and relies on both the mathematical features of the entropy and the specific physical system. Indeed, the $T=0$ block entropies of the $1 / 2$-spin $d=1$ quantum system at criticality are given by $S_{1}(L) \propto \ln L$ (i.e., nonextensive), and $S_{\left[\sqrt{9+c^{2}}-3\right] / c}(L) \propto L$ (i.e., extensive). Moreover, the $T=0$ block entropies of the $d=2$ bosonic system are given by $S_{1}(L) \propto L$ (i.e., nonextensive), and $S_{q}(L) \propto L^{2}$ for special values of $q<1$ (i.e., extensive); for instance, $q_{\text {ent }} \simeq 0.87$ for vanishing gap energy.

More generally, it is known (see Refs. [17, 18] and references therein) that, for $d$-dimensional bosonic systems (e.g., a black hole [19, 20, 21]), $S_{1}$ follows the area law, i.e., $S_{1}(L) \propto L^{d-1}$ (nonextensive). Let us point out that the behavior of the block entropy $S_{1}(L)$ for these quantum systems matches well-known results in conformal field theory (as noted above), where the analogous of the block entropy is the so-called geometric entropy, defined in the continuum [19, 20, 21, 38]. As first suggested by 't Hooft (1985) and later shown by Callan and Wilczek (1994), the geometric entropy is the first quantum correction to a thermodynamical entropy, which reduces to the Bekenstein-Hawking entropy for black holes [19, 20, 21]. A direct connection between entropy and boundary area has been clearly suggested and numerically implemented in Refs. [19, 20] (compare with Refs. [17, 18] and references therein). Finally, the relation among entanglement entropy, the black hole area law and other concepts such as the holographic bound is still an open problem [44]. In this context, it is interesting to note that a logarithmic behavior for $d=1$ and the area law for $d>1$ for a large class of fermionic and bosonic $d$-dimensional many-body Hamiltonians with short-range interaction at $T=0$ can 
be unified through $S_{1}(L) \propto\left[L^{d-1}-1\right] /(d-1) \equiv \ln _{2-d} L$ (i.e., nonextensive, $\ln L$ for $d=1$, and $L^{d-1}$ for $d>1$, area law) 45], which would correspond to a large class (not yet completely identified) of fully entangled quantum systems. For all these systems, one could expect that a value of $q$ exists such that $S_{q}(L) \propto L^{d}$ (i.e., extensive). In this paper our conjecture is verified for $d=1$ quantum spin chains and $d=2$ bosonic systems and is therefore promising also in higher dimensions.

The present work has benefited from early collaboration with D. Rossini and D. Patanè, fruitful discussions with R. Fazio, V. Giovannetti, A. Pluchino, A. Rapisarda, and interesting related comments by G. 't Hooft. It was partially supported by the Centro di Ricerca Ennio De Giorgi of the Scuola Normale Superiore, and by $\mathrm{CNPq}$ and Faperj (Brazilian Agencies).
[1] C. Tsallis, M. Gell-Mann, Y. Sato, Proc. Nat. Acad. Sci. 102, 15377 (2005).

[2] C. Tsallis, J. Stat. Phys. 52, 479 (1988).

[3] C. Tsallis, Entropy, in Encyclopedia of Complexity and Systems Science (Springer, Berlin, in press); C. Tsallis, in Introduction to Nonextensive Statistical Mechanics: Approaching a Complex World (Springer, Berlin, in press).

[4] M. Gell-Mann and C. Tsallis, eds., Nonextensive Entropy: Interdisciplinary Applications (Oxford University Press, New York, 2004).

[5] J.P. Boon and C. Tsallis, eds., Nonextensive Statistical Mechanics: New Trends, New Perspectives, Europhysics News 36, 6 (2005).

[6] J. Havrda and F. Charvat, Kybernetica 3, 30 (1967); Z. Daróczy, Inf. Control 16, 36 (1970); J. Lindhard and V. Nielsen, K. Dan. Vidensk. Selsk. Mat. Fys. Medd. 38 (9), 1 (1971).

[7] S. Umarov, C. Tsallis, and S. Steinberg, Milan J. Math. 76 (2008) [DOI 10.1007/s00032-008-0087-y].

[8] P. Douglas, S. Bergamini, and F. Renzoni, Phys. Rev. Lett. 96, 110601 (2006).

[9] B. Liu and J. Goree, Phys. Rev. Lett. 100, 055003 (2008).

[10] S. Sachdev, Quantum Phase Transitions (Cambridge Univ. Press, Cambridge, 2000).

[11] A. Osterloh, L. Amico, G. Falci, and R. Fazio, Nature 416, 608 (2002).

[12] T.J. Osborne and M.A. Nielsen, Phys. Rev. A 66, 032110 (2002).

[13] G. Vidal, J.I. Latorre, E. Rico, and A. Kitaev, Phys. Rev. Lett. 90, 227902 (2003).

[14] A.R. Its, B.-Q. Jin, and V.E. Korepin, J. Phys. A 38, 2975 (2005).

[15] J.I. Latorre, E. Rico, and G. Vidal, Quant. Inf. and Comp. 4, 48 (2004).

[16] L. Amico, R. Fazio, A. Osterloh, and V. Vedral, Rev. Mod. Phys. 80, 517 (2008).

[17] T. Barthel, M.C. Chung, and U. Schollwock, Phys. Rev. A 74, 022329 (2006).

[18] M.B. Plenio, J. Eisert, J. Dreißig, and M. Cramer, Phys. Rev. Lett. 94, 060503 (2005); M. Cramer, J. Eisert, M.B. Plenio, and J. Dreißig, Phys. Rev. A 73, 012309 (2006).

[19] L. Bombelli, R.K. Koul, J. Lee, and R.D. Sorkin, Phys. Rev. D 34, 373 (1986).

[20] M. Srednicki, Phys. Rev. Lett. 71, 666 (1993).

[21] J.M. Bardeen, B. Carter, S.W. Hawking, Commun. Math. Phys. 31, 161 (1973); J.D. Bekenstein, Lett. Nuovo Cimento 4, 737 (1972); Phys. Rev. D 7, 2333 (1973); G. 't Hooft, Nuclear Physics B 256, 727 (1985); C.G. Callan, F. Wilczek, Phys. Lett. B 333, 55 (1994); D. Kabat, M.J. Strassler, Phys. Lett. B 329, 46 (1994);
J.D. Bekenstein, Contemp. Phys. 45, 31 (2004).

[22] K. Zyczkowski, I. Bengtsson, Ann. Phys. (N.Y.) 295, 115 (2002).

[23] C. Brukner and A. Zeilinger, Phys. Rev. A 63, 022113 (2001).

[24] F. Giraldi, P. Grigolini, Phys. Rev. A 64, 032310 (2001).

[25] C. Tsallis, S. Lloyd, and M. Baranger, Phys. Rev. A 63, 042104 (2001); S. Abe and A.K. Rajagopal, Physica A 289, 157 (2001).

[26] S. Abe and A.K. Rajagopal, Phys. Rev. A 60, 3461 (1999); S. Abe, Phys. Rev. A 65, 052323 (2002).

[27] A.K. Rajagopal and R.W. Rendell, Phys. Rev. A 72, 022322 (2005).

[28] M.B. Plenio, S. Virmani, Quant. Inf. Comp. 7, 1 (2007).

[29] A. Popescu, A.J. Short, and A. Winter, Nature Physics 2, 754 (2006).

[30] F. Mintert, K. Zyczkowski, Phys. Rev. A 69, 022317 (2004).

[31] E. Lieb, T. Schultz, and D. Mattis, Ann. Phys. (N.Y.) 16, 407 (1961).

[32] P. Pfeuty, Ann. Phys. (N.Y.) 57, 79 (1970).

[33] E. Barouch, B.M. McCoy, and M. Dresden, Phys. Rev. A 2, 1075 (1970); E. Barouch and B.M. McCoy, Phys. Rev. A 3, 786 (1971).

[34] F. Caruso and C. Tsallis, in Complexity, Metastability and Nonextensivity, eds. S. Abe, H.J. Herrmann, P. Quarati, A. Rapisarda, and C. Tsallis, American Institute of Physics Conference Proceedings 965 (New York, 2007), pp. 51.

[35] C. Anteneodo and C. Tsallis, Phys. Rev. Lett. 80, 5313 (1998).

[36] A. Pluchino, A. Rapisarda, and C. Tsallis, Eur. Phys. Lett. 80, 26002 (2007).

[37] R. Savit, Rev. Mod. Phys. 52, 453 (1980).

[38] P. Calabrese and J. Cardy, J. Stat. Mech.: Theor.Exp., P06002 (2004); C. Holzhey, F. Larsen, and F. Wilczek, Nucl. Phys. B 424, 443 (1994).

[39] A. Robledo, Molecular Physics 103, 3025 (2005).

[40] B.-Q. Jin, V.E. Korepin, J. Stat. Phys. 116, 79 (2004).

[41] F. Franchini, A.R. Its, and V.E. Korepin, J. Phys. A: Math. Theor. 41, 025302 (2008).

[42] V. Eisler, Ö. Legeza, and Z. Racz, J. Stat. Mech.: Theor. Exp., P11013 (2006).

[43] I. Bediaga, E.M.F. Curado, and J.M. de Miranda, Physica A 286, 156 (2000).

[44] R. Bousso, Rev. Mod. Phys. 74, 825 (2002).

[45] The function $\ln _{q} x \equiv \frac{x^{1-q}-1}{1-q}(x>0 ; q \in \mathcal{R})$ naturally emerges within nonextensive statistical mechanics.

[46] http://tsallis.cat.cbpf.br/biblio.htm 